\title{
Land Cover Classification Accuracy Assessment Using Full-Waveform LiDAR Data
}

\author{
Kuan-Tsung Chang ${ }^{1, *}$, Feng-Chi Yu ${ }^{2}$, Yi Chang ${ }^{3}$, Jin-Tsong Hwang ${ }^{4}$, Jin-King Liu ${ }^{5}$, Wei-Chen Hsu ${ }^{5}$, and \\ Peter Tian-Yuan Shih ${ }^{6}$ \\ ${ }^{l}$ Department of Civil Engineering and Environmental Informatics, Minghsin University of Science and Technology, Hsinchu, \\ Taiwan, R.O.C. \\ ${ }^{2}$ Institute of Service Industries and Management, Minghsin University of Science and Technology, Hsinchu, Taiwan, R.O.C. \\ ${ }^{3}$ Institute of Ocean Technology and Marine Affairs, National Cheng Kung University, Tainan, Taiwan, R.O.C. \\ ${ }^{4}$ Department of Real Estate and Built Environment, College of Public Affairs, National Taipei University, New Taipei City, \\ Taiwan, R.O.C. \\ ${ }^{5}$ LiDAR Technology Co., Hsinchu, Taiwan, R.O.C. \\ ${ }^{6}$ Department of Civil Engineering, National Chiao-Tung University, Hsinchu, Taiwan, R.O.C.
}

Received 29 December 2013, revised 8 June 2014, accepted 2 December 2014

\begin{abstract}
The geomorphology of Taiwan is characterized by marked changes in terrain, geological fractures, and frequent natural disasters. Because of sustained economic growth, urbanization and land development, the land cover in Taiwan has undergone frequent use changes. Among the various technologies for monitoring changes in land cover, remote sensing technologies, such as LiDAR, are efficient tools for collecting a broad range of spectral and spatial data. Two types of airborne LiDAR systems exist; full-waveform (FW) LiDAR and traditional discrete-echo LiDAR. Because reflected waveforms are affected by the land object material type and properties, the waveform features can be applied to analyze the characteristics specifically associated with land-cover classification (LCC). Five types of land cover that characterize the volcanic Guishan Island were investigated. The automatic LCC method was used to elucidate the spectral, geomorphometric and textural characteristics. Interpretation keys accompanied by additional information were extracted from the FW LiDAR data for subsequent statistical and separation analyses. The results show that the Gabor texture and geomorphometric features, such as the normalized digital surface model (nDSM) and slopes can enhance the overall LCC accuracy to higher than 90\%. Moreover, both the producer and user accuracy can be higher than $92 \%$ for forest and built-up types using amplitude and pulse width. Although the waveform characteristics did not perform as well as anticipated due to the waveform data sampling rate, the data provides suitable training samples for testing the waveform feature effects.
\end{abstract}

Key words: Land cover, Classification, Geomorphometric, Waveform, Texture

Citation: Chang, K. T., F. C. Yu, Y. Chang, J. T. Hwang, J. K. Liu, W. C. Hsu, and P. T. Y. Shih, 2015: Land cover classification accuracy assessment using full-waveform LiDAR data. Terr. Atmos. Ocean. Sci., 26, 169-181, doi: 10.3319/TAO.2014.12.02.02(EOSI)

\section{INTRODUCTION}

Land cover is the physical material on the Earth's surface, including grass, asphalt, trees, bare ground, and water. Although these terms are frequently used interchangeably, land cover is distinct from land use, which refers to how people use the land for socioeconomic activities (Comber et al. 2005). Changes in land use and land cover (LULC) are the critical driving forces of change in atmospheric,

\footnotetext{
* Corresponding author

E-mail:ktchang@must.edu.tw
}

climatic, and ecological systems (Feddema et al. 2005; Pielke 2005). Changes in LULC occur because of natural hazards such as typhoons and earthquakes, and human activities such as deforestation. Taiwan comprises a land area of approximately $36000 \mathrm{~m}^{2}$, featuring plains (26.68\%), hills $(27.31 \%)$, and mountains $(46.01 \%)$. The mountainous regions encompass high mountain valleys with deep and fault-knit environments, fragile geological features, abrupt slopes and steep rivers. According to the National Fire Agency (2007), 270 natural disaster events occurred 
in Taiwan from 1958 - 2007, including typhoons (71.1\%), floods (15.0\%), and earthquakes (8.5\%). Strong, sustained economic growth has driven urbanization and land development, causing frequent changes in the types of LULC. Compounding the rapid development effects, these considerable LULC changes have coincided with the destruction of natural environments and the improper use of soil and water resources. Thus, extant land-cover information should be updated promptly, efficiently and continually. Accurate land-cover mapping provides crucial information that facilitates resource management, planning and intergovernmental cooperation for addressing global warming and minimizing biodiversity reduction. Traditional methods for land-cover mapping include photogrammetry and remote sensing. The types of sensors used for land-cover mapping are passive (e.g., optical satellite or digital camera), active (e.g., radar or LiDAR), or a combination of both. Previous aerial survey payload sensors have been equipped with film-type aerial cameras; however, these have been superseded by metric digital cameras. In addition to capturing images comprised of red, green, and blue (RGB) bands in the visible spectra, metric digital cameras can capture images in the near-infrared (NIR) band. The advantages of employing remote sensing technology to examine LULC include extensive range, immediacy and periodicity of the obtainable information. Remote sensing technology can be applied to monitor current changes in LULC (Chang et al. 2012b).

A LiDAR system is comprised of multiple subsystems; namely, a global positioning system (GPS), inertial measurement unit and laser scanner. Currently, the high volumes of spatial data collected within a short period using LiDAR systems are typically used for quantitative analyses and modeling in geology, coastal erosion and geomorphology studies. Airborne LiDAR technology can be used to collect multiple laser returns at pulse repetition rates of up to $500 \mathrm{KHz}$. The positional accuracy of the resultant laser pulse return is typically at the decimeter level. Thus, the obtained standard airborne LiDAR survey products include all points, ground points, digital surface models (DSMs) and digital elevation models (DEMs). Two types of airborne LiDAR system are currently available; full-waveform $(\mathrm{FW})$ and discrete-echo LiDAR. The return signal in discrete echo LiDAR systems is filtered to export multiple echoes. For each transmitted laser pulse only three to seven echoes are typically used to record the intensity and three-dimensional coordinates. However, FW LiDAR systems can record the entire waveform for each transmitted laser pulse. A waveform typically involves responses per nano-second, with a maximum of 255 echoes obtained (Fig. 1); permitting an exceptionally high level of information to be preserved. Additional land information and high-density point-cloud data can be acquired to improve the accuracy of digital terrain models (DTMs) (Mücke 2008; Wagner et al. 2008). Because the reflected waveforms are affected by the material type and properties of detected land objects, the waveform features can be applied to analyze the physical characteristics associated with land-cover classification (LCC) (Neuenschwander et al. 2009). LiDAR systems have been used in close-range or aerial topographical surveys to classify vegetation in forested areas and map disasters (Knabenschuh and Petzold 1999; Wehr and Lohr 1999; Thiel and Wehr 2001; Beraldin et al. 2010).

Three frequently used methods for surveying the status of land cover are ground aerial and space borne surveys (Lillesand et al. 2004; Liu et al. 2009) or a combination of these methods (Galli et al. 2008). The ground survey method was first employed in Taiwan for land-use censuses between 1993 - 1995 (Huang et al. 2007). Ground surveys are slow but highly accurate. When an inventory area is large accessibility tends to be low. Therefore, neither near-real-time

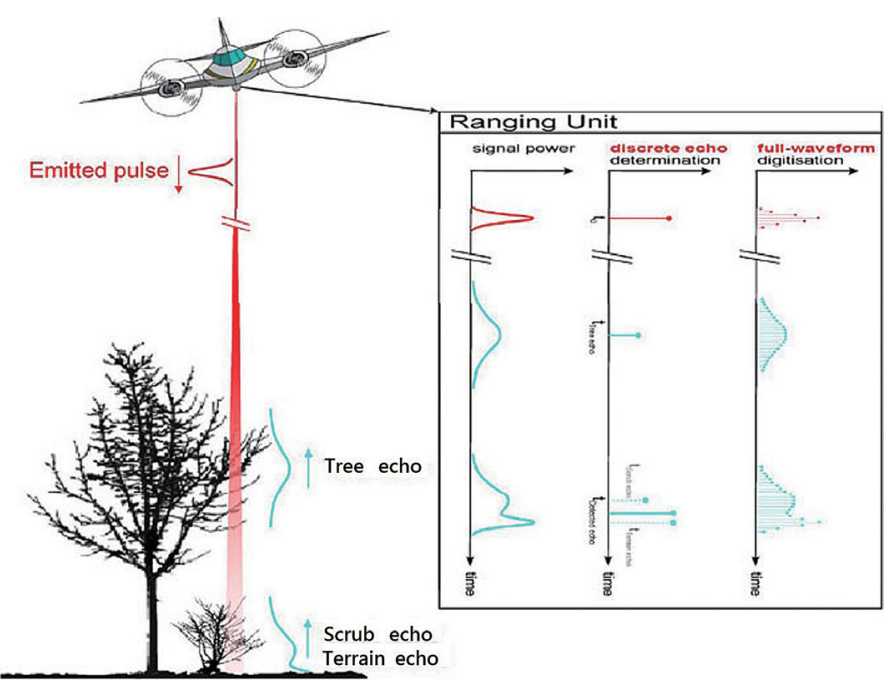

Fig. 1. Full-waveform (FW) LiDAR system return signal (Doneus et al. 2008). 
surveying nor complete coverage is possible. Conversely, photographic or image interpretation approaches are not subject to accessibility problems. Aerial photography and high special resolution stereoscopic images have been used frequently to characterize geometric boundaries and attributes. These approaches can be used to resolve and clearly define individual land-cover objects. Manual, semiautomatic and automatic interpretations of photographic data have been used. Manual interpretation requires adequately trained personnel to delineate land-cover objects from stereoscopic images or orthorectified images. However, the conventional approach for interpreting photographic images is time consuming and labor intensive (Lillesand et al. 2004; Huang et al. 2007). Automatic LCC is based on certain criteria and algorithms (i.e., image classification methods) that are advantageous because of their objectivity. Several researchers have attempted to identify patterns or objects by applying pixel-based classification methods such as the maximum likelihood (ML) method, artificial neural networks (ANNs) (Zurada 1992; Barlow et al. 2003; Chang and Liu 2004), and support vector machines (SVMs) (Zhu and Blumberg 2002; Foody and Mathur 2004; Camps-Valls and Bruzzone 2005; Chang et al. 2010). Traditional methods for classifying remote sensing data are based on statistical analyses (e.g., the ML method) and priori statistical information (e.g., probability distributions). According to the Hughes effect, increasing the number of data bands requires using additional training samples; otherwise, the classification criteria performance is degraded. ANNs are both powerful and versatile computational tools for organizing and correlating information to solve problems that are exceedingly complex, poorly understood or excessively resource intensive. However, a trained network is a black box, in which the internal workings of the network are unknown. The derived solution is typically a local extreme value (Bischof et al. 1992). SVMs are novel pattern recognition models based on adaptive learning methods. An SVM can be employed to process high-dimensional data and these models have become increasingly used in applications involving remote sensing. Some SVM models have been used in LULC classification projects, the experimental results of which showed that SVM models are more accurate compared with alternative supervised classification methods (Chang et al. 2012a).

This study therefore used an SVM model integrating spectral, geomorphometric, waveform, and textural features to conduct LCC. Geomorphometric features were generated from a LiDAR-based DEM and DSM. Five types of land cover that characterize Guishan Island, a volcanic island in eastern Taiwan, were used in an experiment to perform accuracy assessment on LCC. Moreover, the interpretation keys with additional information that were extracted from FW LiDAR (e.g., amplitude and pulse width) were also evaluated to determine the relationship with various types of land cover.

\section{METHODOLOGY}

Orthorectified aerial images in the NIR band and FW LiDAR data were employed in this study to extract spectral features [i.e., the normalized differential vegetation index (NDVI)] and greenness index), geomorphometric features [i.e., the normalized digital surface model (nDSM)], slope, and aspect), waveform features from the LiDAR data [i.e., amplitude and pulse width (Lin and Mills 2010)], and their corresponding textural features (i.e., variance, entropy, and Gabor filter). The nDSM obtained by subtracting the DSM and DEM entails the normalized height of objects above the bare ground surface. Subsequently, an SVM method was employed to assess the LCC accuracy. The waveform patterns for various types of land cover were characterized using a statistical method. The method for extracting the waveform and Gabor texture features and the principle of the SVM model implemented in this study are detailed in the following section. For further details regarding the other LiDAR-derived features and classifications, please refer to Chang et al. (2010, 2012a)

\subsection{Extraction of Waveform and Texture Features}

\subsubsection{Waveform Features}

To ensure that the sample data fit the function model waveform decomposition methods for obtaining $\mathrm{FW} \mathrm{Li-}$ DAR data assume that a return pulse is a distribution function model. The most frequently used models form a symmetric distribution (e.g., Gaussian) (Wagner et al. 2006), asymmetric Weibull function (Jutzi and Stilla 2006), or log-normal distribution (Liao 2013). Point-cloud data (LAS 1.3 file format) were imported into the Terra Scan software version 009.014 in this study (Terrasolid, Finland). The process for extracting and exporting the waveform data as an ASCII text file required using the waveform function. The amplitude and pulse width were then derived using the Gaussian decomposition method. Except for the two mentioned waveform features, a normalized amplitude feature obtained based on the amplitude value divided by the total amount of amplitude was calculated as a waveform feature to reduce the gain offset effects.

\subsubsection{Gabor Texture Feature}

The Gabor filter, which is a linear filter with an impulse response defined according to a harmonic function multiplied by a Gaussian function, is ideally localized based on the uncertainty principle in both spatial and frequency domains. Thus, a Gabor filter can be highly selective in both position and frequency in enhancing the texture boundaries detection (Vyas and Rege 2006). The Gabor filter-related segmentation paradigm is based on a filter bank model, in which several filters are applied simultaneously to an input 
image. The filters focus on a specific range of frequencies. If an input image contains two unique texture areas, the local frequency difference between the areas is used to detect the textures in one or more filter output sub images.

Gabor filters can be configured to have various shapes, bandwidths, center frequencies and orientations by adjusting the corresponding parameters. The filter can thus be configured to pass any spatial frequency elliptical region.

$$
\begin{aligned}
& g(x, y)=\frac{1}{2 \pi \sigma_{x} \sigma_{y}} \exp \left[-\frac{1}{2}\left(\frac{\overline{x^{2}}}{\sigma_{x}}+\frac{\overline{y^{2}}}{\sigma_{y}}\right)\right] \cos \left[2 \pi j \frac{\bar{x}}{\lambda}\right] \\
& \bar{x}=x \cos \theta+y \sin \theta \\
& \bar{y}=-x \sin \theta+y \cos \theta
\end{aligned}
$$

In Eq. (1), $\sigma_{x}$ and $\sigma_{y}$ characterize the spatial extent and bandwidth of the filter for determining the effective size of neighboring pixels for the weighted summation. $\theta$ specifies the orientation of the Gabor filters and $\lambda$ is the wavelength at which the $\operatorname{cosine}$ factor $\cos (2 \pi \bar{x} / \lambda)$ determines the preferred spatial frequency $1 / \lambda$ of the receptive field function $g(x, y)$. A filter exhibits a strong response to an edge with a normal parallel to the orientation $\theta$ of the sinusoid. In 1946 Gabor proposed expanding a wave based on Gaussian wave packets (e.g., a sine wave multiplied by a Gaussian function). If a signal is modulated by a Gaussian window of a certain width and central time, a Fourier expansion of the modulated signal then returns a measure of the local spectrum. Although such a spectrum is not unique because the width of the Gaussian window is arbitrary, local spectra are highly useful. If a collection of local spectra is computed for a series of window positions, the result is the time-frequency decomposition known as a Gabor transformation. When two textures differ in a given image a Gabor filter is configured to produce a step change in the output $O(x, y)$ at the texture boundaries (Hwang et al. 2011). A Matlab code was applied in this study to generate the Gabor texture image. After applying a trial-and-error approach we applied 0.2 or 0.1 for $f$, and 2.0 for $\sigma_{x}$ and $\sigma_{y}$. The optimal texture image was derived using a window size with the filter set at $9 \times 9$ with a zero orientation angle.

\subsection{SVMs}

The SVM was proposed by Vapnik to address the problems of pattern classification and nonlinear regression by minimizing the structural risk (Vapnik 1998). Based on the structural minimization risk principle in computational learning theory, SVMs are designed to identify the separating hyper plane based on the maximal margin to differentiate between positive and negative samples from a training set. The simplest type of SVM is a binary classifier, which determines whether an input image belongs to one of two classes. The SVM requires a set of training samples com- prising positive and negative samples to produce an SVM and the corresponding Class C. Positive samples belong to Class C. After the images are preprocessed all samples are translated into $n$-dimensional vectors.

There are two types of multiclass SVM systems; oneversus-all and one-versus-one. The one-versus-all SVM must train $k$ binary SVMs, where $k$ is the number of classes. The $i$ th SVM is trained using all samples of the $i$ th class as positive samples and the remaining samples as negative. After all SVMs are established using positive and negative samples, the system trains all $k$ SVMs. Thus, the SVM builds $k$ decision functions. The decision values are computed by the decision functions, and the maximal value and corresponding class in Eq. (2) are used as the resulting class to test the data.

Class of $x=\arg \max _{i=1 \ldots k}\left[\left(w^{i}\right)^{T} \cdot \varphi(x)+b^{i}\right]$

Where $\varphi(x)$ is a mapping function in mapping training data $x_{i}$ to high-dimensional space, $w$ is a vector normal to the hyper plane, and $b$ is the bias. By contrast, the oneagainst-one SVM trains a corresponding $\mathrm{SVM}_{i j}$ for every combination of two classes (i.e., $i$ and $j$ ). It therefore trains $k \times(k-1) / 2 \mathrm{SVMs}$ to build $k \times(k-1) / 2$ decision functions. For each input object all of the decision values are computed and a voting algorithm is applied to determine which class the object belongs to. If $\operatorname{sign}\left(w_{i j} x+b_{i j}\right)$ shows that $x$ belongs to the $i$ th class, then the vote count for that class increases by one; otherwise, the vote count for the $j$ th class increases by one. Finally, $x$ is predicted to be the class with the most votes. The LibSVM function in WEKA (version 3.6.1) was employed for the SVM classification in this study.

\section{CASE STUDY}

Two experiments were conducted in this study: experiment 1 was designed to characterize the types of land cover based on the FW LiDAR pattern, and experiment 2 was designed to assess the LCC accuracy based on various combinations of features. Experiment 1 was conducted to determine whether the waveform information could be applied to improve land-cover recognition. A river channel named the Taimali river corridor in Eastern Taiwan was surveyed using FW LiDAR in 2012 as the study area for experiment 1. The amplitude and pulse width of the waveforms were extracted as the primary features for analyzing various types of land cover. Statistical analysis was conducted to characterize the significant features of various land-cover types. The eight land-cover types analyzed in this study were forest, wet sand, dry sand, asphalt, cement houses, grass, metal houses, and other artificial structures (Fig. 2). In addition to the mean and standard deviation of the amplitude and pulse width data, the nDSM and slope of DTM were calculated to 
differentiate among the various types of land cover.

A separation measure was developed to indicate similar clusters that were assumed to exhibit data density as a decreasing function of the distance from the cluster vector. The measure was applied to infer the appropriateness of the data partitions for comparing the relative appropriateness of various data divisions. The measure depended on neither the number of analyzed clusters nor the method for partitioning the data. Furthermore, it could be used to guide a cluster-seeking algorithm. The four separation measures were divergence, transformed divergence (TD), Bhattacharyya distance (BD), and JM distance (JD). These separation measures were performed using the SEPSIG function of Idrisi Taiga software (Clark Labs, USA). SEPSIG produced a table encompassing several sections, each detailing the separability among the band combinations of a specific pairwise combination of signatures. Ultimately, an additional section detailed the mean separability for the entire signature group (calculated as the mean of all pairwise combinations). Within each section the left column specified all possible subset combinations for the total number of bands, while the right column specified the calculated value for the applied separability measure. A multiplier was applied to scale the TD measure into a useful range. Typical multipliers (2100 or 2000) were used (default value 2000), and the TD measure was applied as the measurement index for validating the degree of discrimination between the defined signatures.

Guishan Island was the study area in experiment 2 (Fig. 3). The island is the top of an andesitic stratovolcano based on the sea floor, exhibiting active fumaroles and solfataras. It has an area of $2.841 \mathrm{~km}^{2}$ and its peak is $401 \mathrm{~m}$ above sea level. The data set was captured using a Riegl LMS680i airborne LiDAR system (altitude, 1400 m; scanning frequency, $270 \mathrm{Khz}$ ) on 7 July 2011.

Orthophotos (spatial resolution, $10 \mathrm{~cm}$ ) were generated from aerial photographs that were captured using direct georeferencing, and orthorectified using LiDAR DSM without employing the ground control points. The NDVI was applied to normalize the adopted orthoimages, which were originally captured in the NIR band (Fig. 4a). The greenness index was calculated based on the spectral features. Certain geomorphometric features (e.g., the slope and nDSM) shown in Fig. 4b were applied in these experiments and their corresponding textural features (e.g., variance, entropy, and Gabor texture) were tested. Figure 4c shows a Gabor texture image generated from the aerial image.

The five types of land cover analyzed in this study area were broadleaf forest (code 1), grassy land (code 2), bare land (code 3), cement pavement (code 4), and built-up area (code 5). Figure 4d shows the ground truth (GT) by

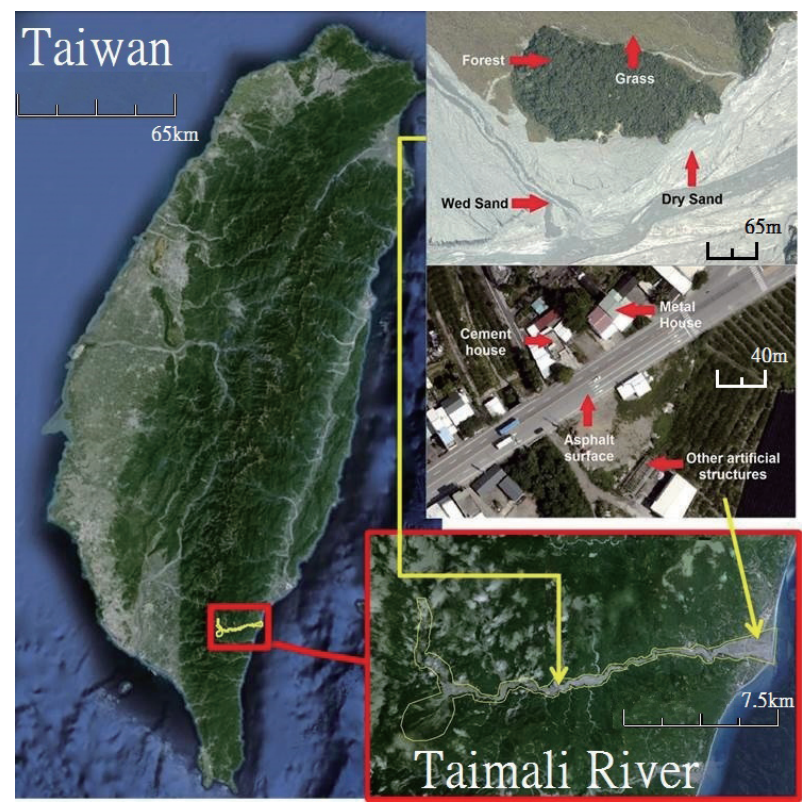

Fig. 2. The eight land-cover types defined for the first study area.

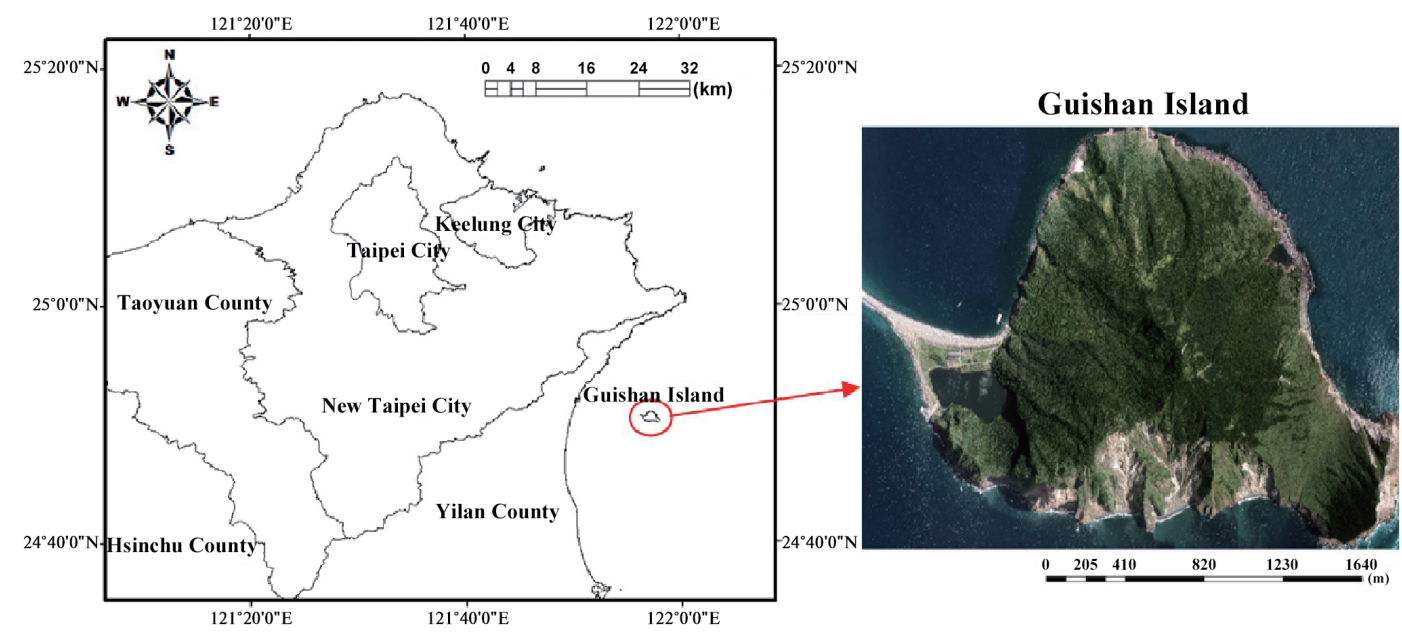

Fig. 3. The second study area : Guishan Island. 
(a)

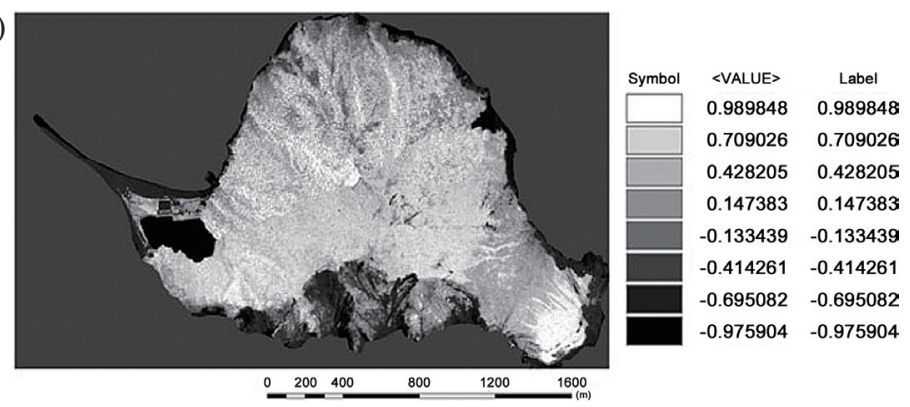

(b)

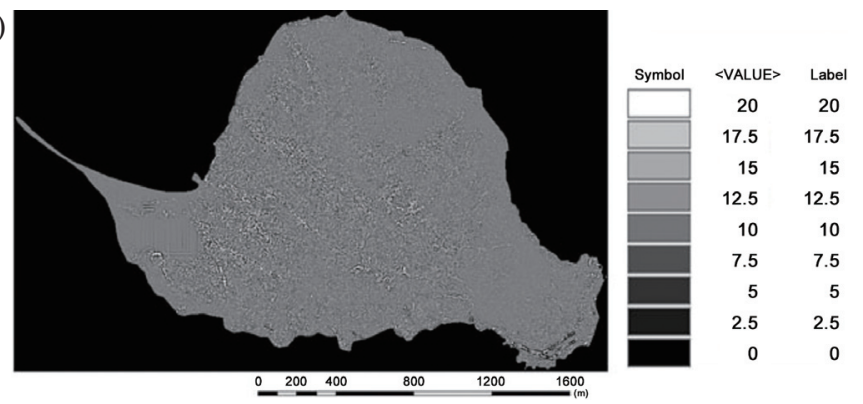

(c)

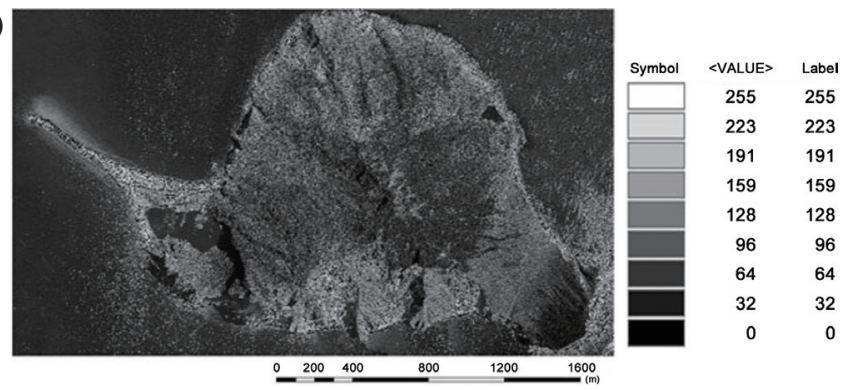

(d)

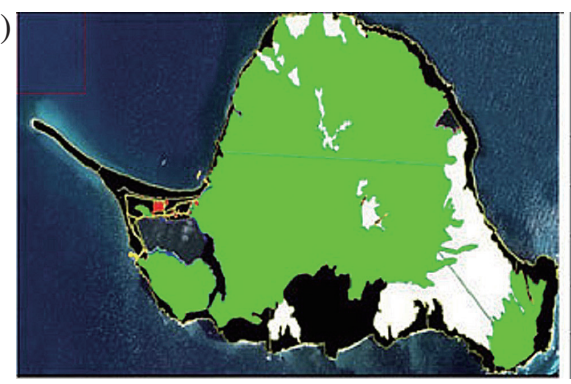

\begin{tabular}{|c|c|c|c|c|} 
CODE & Color & Land cover types & $\begin{array}{c}\text { Training } \\
\text { samples } \\
\text { (pixels) }\end{array}$ & $\begin{array}{c}\text { Total } \\
\text { amount } \\
\text { (pixels) }\end{array}$ \\
\hline 1 & & Broadleaf Forest & 1200 & 1648391 \\
\hline 2 & & Grass land & 1050 & 392848 \\
\hline 3 & & Bare land & 620 & 520507 \\
\hline 4 & & Cement pavement & 273 & 10676 \\
\hline 5 & & Built-up area & 658 & 4466 \\
\hline
\end{tabular}

Fig. 4. Partial used experimental data and ground truth in the second experiment. (a) NDVI image; (b) nDSM; (c) Gabor texture for the aerial image; (d) Ground Truth.

combining the manual interpretation result from high-resolution orthophotos and the second national land-use investigation result in 2006 (Huang et al. 2007).

The experimental data were first imported into ENVI 5.x (Exelis Visual Information Solutions, USA) to define the test regions for each type of land cover (ITT 2010). The processed image was subsequently overlapped with the GT image in ArcGIS 9.3 (ESRI, USA) to summarize the statistical values for the spectral, geomorphometric, waveform and corresponding textural features within the test regions. The attributes and their corresponding codes were then exported as a data table, which was imported into WEKA as the training and testing data set. The accuracy of the pixelbased SVM classification results was then assessed.

\section{RESULTS AND DISCUSSION}

\subsection{Characterizing Waveform Patterns for Various Land-Cover Types}

Numerous studies have confirmed that waveform data can yield land-cover information (Bretar et al. 2009; Neuenschwander et al. 2009; Alexander et al. 2010; Mallet et al. 2011), and additional high density point-cloud data can be acquired to improve DTM accuracy (Mücke 2008; 
Liao 2013). In addition, because the reflected waveforms are affected by the type of material and properties of detected land objects, the waveform features (e.g., amplitude and echo width) can be applied to analyze specific characteristics associated with LCC.

The waveform data extracted from certain LiDAR points located in proximity to each land-cover type were obtained using the waveform function in the Terra Scan software to confirm whether the actual types of land cover were in agreement with specific waveform patterns. The full waveform plots for the sample points on the interested regions for the broadleaf forest, three man-made structures, asphalt pavement, wet sand and dry sand are shown in Figs. 5a - c, respectively. The horizontal axis is returned pulse and the vertical axis is amplitude in Figs. 5a - c. As shown in Fig. 5a, waveforms for three sample points of the broadleaf forest type consisted of laser shots with multiple echoes (Mücke 2008). This waveform characteristic should be affected by the canopy structure. However, only one major pulse occurred among the waveforms for the remaining types of land cover, as shown in Figs. 5b and c. Therefore, the number of pulses may be among the crucial features to discriminate the forest with other types of land cover in the experiment.

Table 1 lists the results from the features statistical analysis for the eight types of land cover. The descriptive statistical measures, such as normality (including mean and standard deviation), were performed using 30 point samples for each type. Because laser shots caused multiple echoes in the class forest, the echo with the maximum amplitude was used to calculate the mean and standard deviation for the corresponding echo width. Table 1 shows that the low processing accuracy of the point-cloud data filtering resulted in increased standard deviations among the nDSM and slope features of the forested areas. A similar amplitude and echo width trend is also apparent. Furthermore, the forest echo amplitudes are more scattered than those of the forest terrain echoes.

The statistical results for the wet sand amplitude values compared with dry sand indicate that the moisture content might have affected the waveform amplitude by weakening the reflection intensity. The amplitude increased when the signals were reflected by dry materials such as cement and metal houses or other man-made structures. The lower value distribution in the amplitude for the wet sand is due to the laser energy absorbed by the aqueous material. This inference should be further validated based on in situ moisture measures.

The statistical echo-width data results for the analyzed land-cover types differed from that of the amplitude; specifically, the echo-width values increased in areas that were characterized by comparatively low water content. For example, the echo width was highest in grassy regions and areas featuring metal housing or other artificial structures (Chen et al. 2013).

Figure 6 shows a scatter diagram plotted by the aver- age and standard deviation amplitude and pulse width values for the eight land-cover types shown in Table 1. The figure shows overlapping values for the grass and artificial structure waveform features, as well as for those of dry sand and asphalt surface. The samples for the class forest could contain different tree species; hence, the standard deviation amplitude and echo width values in the class forest are higher than those for other land-cover types. An overlapping phenomenon also occurs in the broad-leaf forest and cement structure in this figure. These results indicate that the waveform features alone are ineffective for distinguishing the aforementioned land-cover types. However, the waveform

(a)

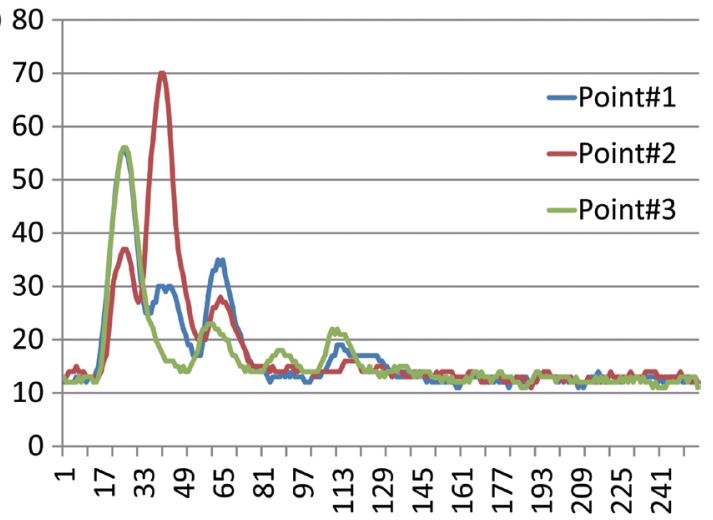

(b)

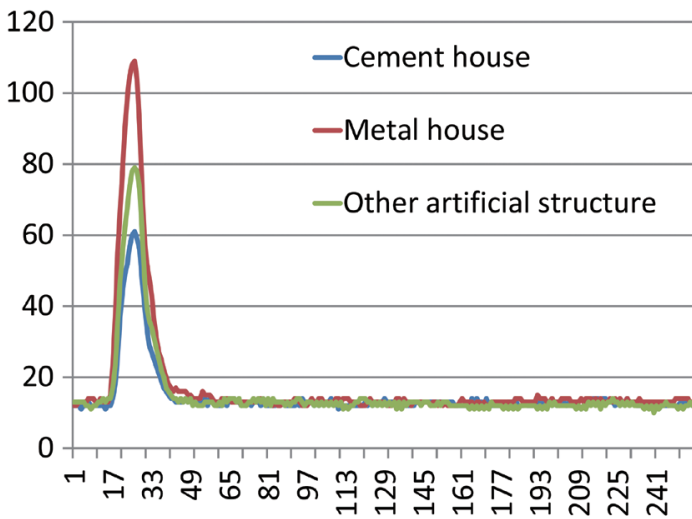

(c)

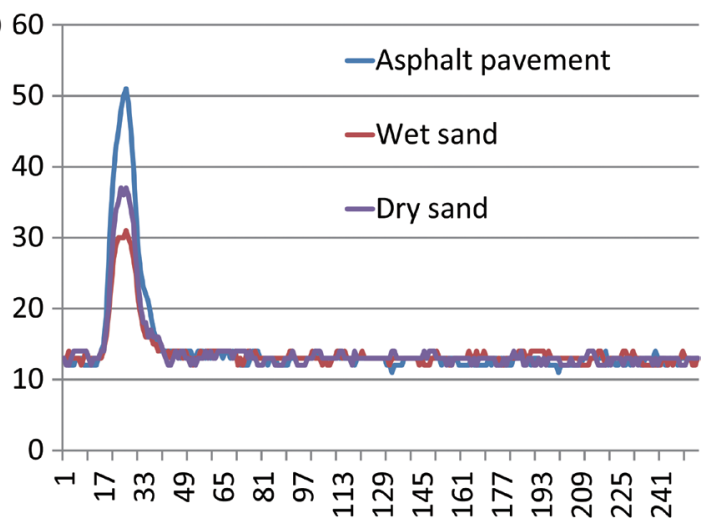

Fig. 5. The waveform pattern for seven land cover types. (a) Broadleaf forest; (b) three structure objects; (c) asphalt, wet and dry sand. 
Table 1. Statistical results for eight land-cover types.

\begin{tabular}{c|cc|cc|cc|cc}
\hline \multirow{2}{*}{ Land cover type } & \multicolumn{2}{|c|}{ Amplitude $(\mathbf{D N})$} & \multicolumn{2}{c|}{ Echo width $(\mathbf{n s})$} & \multicolumn{2}{c|}{ nDSM $(\mathbf{m})$} & \multicolumn{2}{c}{ Slope (degree) } \\
\cline { 2 - 9 } & Average & STDEV & Average & STDEV & Average & STDEV & Average & STDEV \\
\hline Forest & 63.6 & 16.1 & 15.9 & 3.91 & 14.829 & 4.153 & 32.389 & 6.864 \\
Wet sand & 30.47 & 5.34 & 8.53 & 2.37 & 0.195 & 0.186 & 1.672 & 0.238 \\
Dry sand & 48.6 & 13 & 14.5 & 2.71 & 0.031 & 0.159 & 1.062 & 0.532 \\
Asphalt surface & 45.3 & 8.27 & 13.67 & 1.91 & -0.005 & 0.021 & 1.179 & 1.171 \\
Cement house & 59.8 & 7.94 & 16 & 1.31 & 5.677 & 1.987 & 4.948 & 0.81 \\
Grass & 83.97 & 16.53 & 20.03 & 2.9 & 0.088 & 0.168 & 4.834 & 4.304 \\
Metal house & 105 & 5.9 & 20.38 & 0.49 & 5.791 & 1.69 & 4.421 & 2.012 \\
Other artificial structure & 88.83 & 8.6 & 19.33 & 0.85 & 0.033 & 0.044 & 1.49 & 0.666 \\
\hline
\end{tabular}

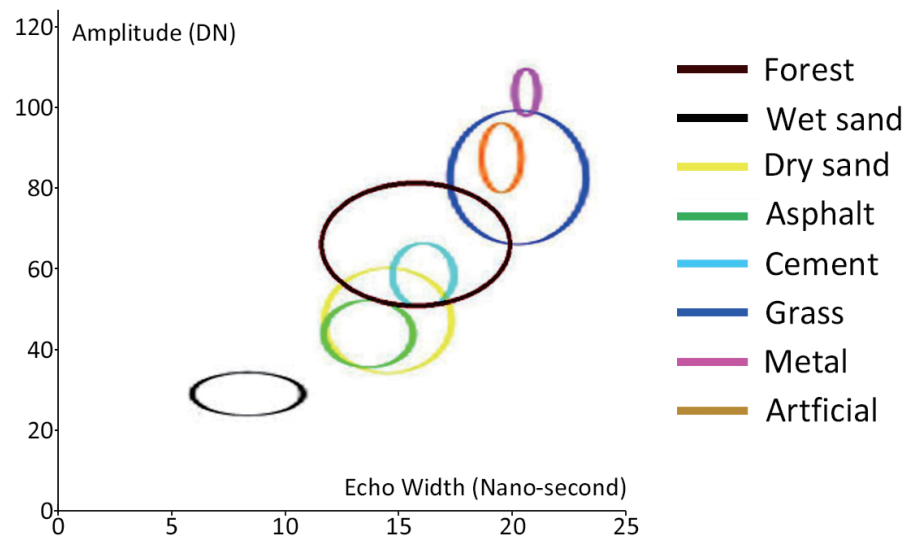

Fig. 6. The scatter diagram of waveform features for eight land cover types.

features have the potential to distinguish asphalt, cement structures, metal structures and wet sand as their clusters in different zones (Fig. 6). Table 2 lists the TD results after the pairwise signature separability combinations were determined. The dry and wet sand land-cover types were the only pair of land-cover types in which the TD value was less than 1900. However, all pairs of land-cover signatures achieved acceptable separation.

\subsection{Accuracy Assessment on LCC}

Experiment 2 was designed to evaluate the effect of applied features on LCC accuracy. Based on the RGB bands in the orthophotos, the combination was gradually increased with the mentioned four kinds of features (e.g., NDVI, geomorphometric features, textural features, and waveform features) in the LCC. Table 3 lists the various combinations of features examined in the LCC experiment accuracy assessment after a trial-and-error test. In these combinations changes in land use may have been affected by the slope and aspect features. Therefore, these two parameters were also incorporated in the LCC experiment. The overall accuracy of these two feature sets is not significantly different from those derived from other feature sets.

Figure 7 shows the overall accuracy of the LCC based on various combinations of features and training samples of various sizes. The "1/3_training samples" represent onethird of all samples randomly chosen as a training data set, and the others were used as a test data set for the accuracy assessment. The "1/2_training samples" represent half of the samples used in a training data set. Sets 22 and 23 yielded the highest overall accuracy levels (> 90\%). Sets $16-17$ and 20 - 21 contained comparatively few features, yielding acceptable classification accuracy and indicating that the presence of geomorphometric features is a crucial factor for obtaining accurate LCC. Regarding the waveform features the normalized amplitudes were more accurate than the other waveform features (based on the results for sets $13-15$ ). The Gabor texture was one of the most accurate textural features for LCC in this study (based on the results for set 12).

Figures $8 \mathrm{a}$ - e show the producer accuracy (PA) results and Figs. 9a - e show the user accuracy (UA) results for each type of land cover based on various combinations of features and training samples of various sizes. Regarding the 
Table 2. A measure result of signatures separation (TD value).

\begin{tabular}{|c|c|c|c|c|c|c|c|c|}
\hline Transformed divergence & Forest & Wet sand & Dry sand & Asphalt surface & Cement house & Grass & Metal house & Other artificial structure \\
\hline \multicolumn{9}{|l|}{ Forest } \\
\hline Wet sand & 2000 & & & & & & & \\
\hline Dry sand & 2000 & 1853.33 & & & & & & \\
\hline Asphalt surface & 2000 & 2000 & 1972.88 & & & & & \\
\hline Cement house & 2000 & 2000 & 2000 & 2000 & & & & \\
\hline Grass & 2000 & 2000 & 1999.56 & 1999.9 & 2000 & & & \\
\hline Metal house & 2000 & 2000 & 2000 & 2000 & 1999.99 & 2000 & & \\
\hline Other artificial structure & 2000 & 2000 & 1992.11 & 1998.82 & 2000 & 1994.03 & 2000 & \\
\hline
\end{tabular}

Table 3. Combination of used features.

\begin{tabular}{l:l|l:l}
\hline Set id & content of features & Set id & \multicolumn{1}{c}{ content of features } \\
\hline Set 1 & RGB & Set 13 & RGB + Pulse Width \\
Set 2 & RGB + Greenees & Set 14 & RGB + Amplitude \\
Set 3 & RGB + NDVI & Set 15 & RGB + normalized Amplitude \\
Set 4 & RGB + DEM & Set 16 & RGB + DEM_Geomorphology \\
Set 5 & RGB + nDSM & Set 17 & RGB + DSM_Geomorphology \\
Set 6 & RGB + DEM_Aspect & Set 18 & RGB + Waveform features (Amplitude + Pulse width) \\
Set 7 & RGB + DEM_Slope & Set 19 & RGB + Waveform features (normalized Amplitude + Pulse Width) \\
Set 8 & RGB + DEM_Entropy & Set 20 & Spectrum + DEM_Geomorphology + Waveform \\
Set 9 & RGB + DEM_Variance & Set 21 & Spectrum + DSM_Geomorphology + Waveform \\
Set 10 & RGB + DSM_Entropy & Set 22 & Spectrum + DEM_Geomorphology + Waveform(nAmp + Pulse Width) \\
Set 11 & RGB + DSM_Variance & Set 23 & Spectrum + DSM_Geomorphology + Waveform(nAmp + Pulse Width) \\
Set 12 & RGB + Gabor texture & & \\
\hline
\end{tabular}

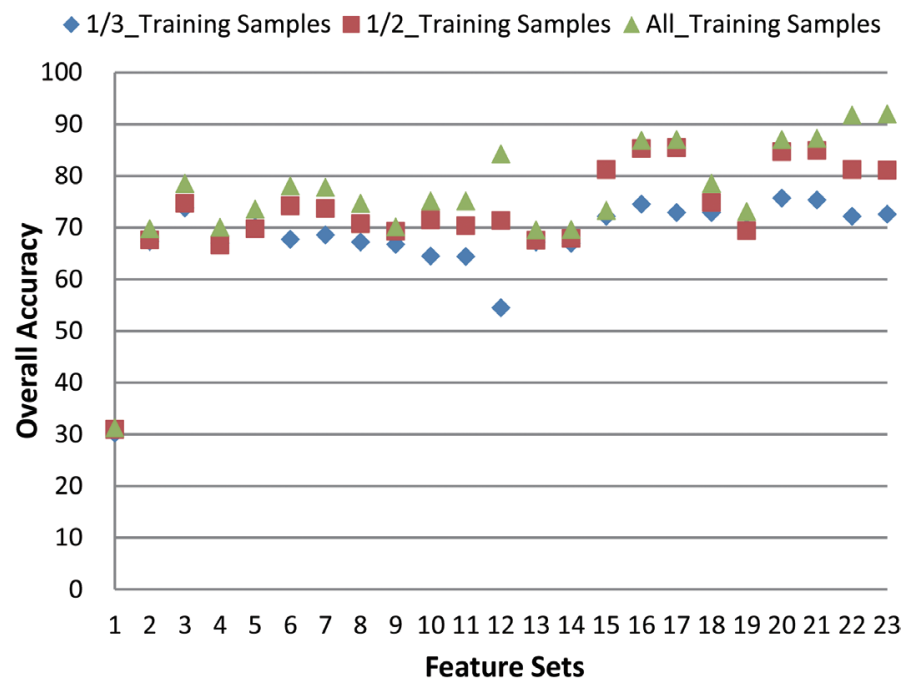

Fig. 7. The land-cover classification (LCC) overall accuracy under different combination of features. 
(a) 1/3_Training Samples $\square$ 1/2_Training Samples $\Delta$ All_Training Samples

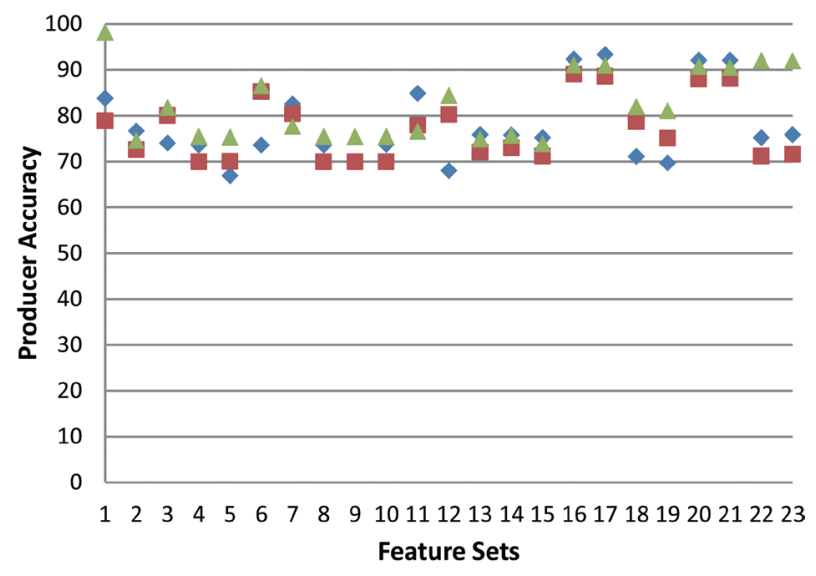

(c) $1 / 3_{-}$Training Samples $\square$ 1/2_Training Samples $\Delta$ All_Training Samples

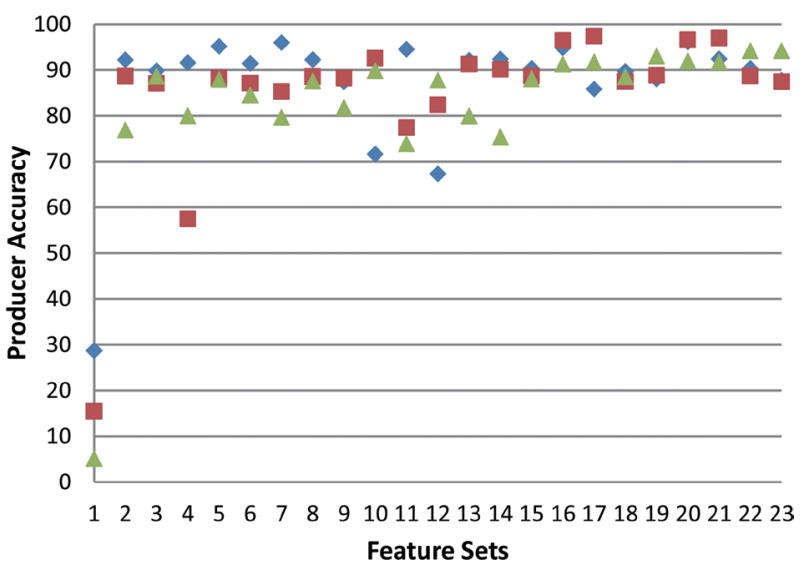

(e) 1/3_Training Samples $1 / 2$ _ Training Samples $\triangle$ All_Training Samples

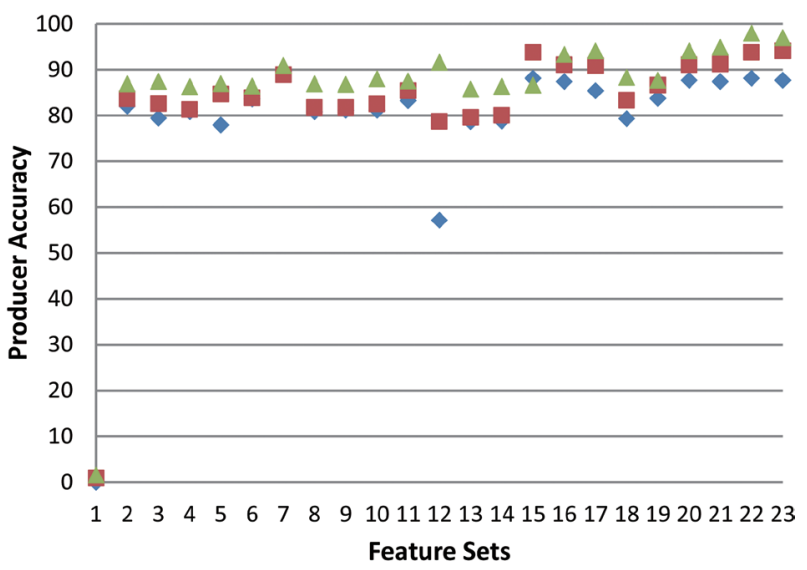

(b) $1 / 3$ _ Training Samples $₫ 1 / 2$ _Training Samples $\Delta$ All_Training Samples

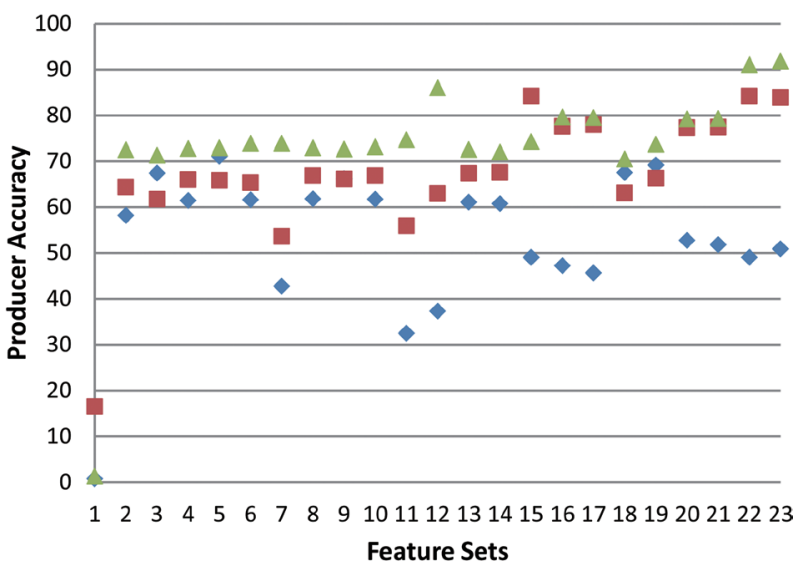

(d) 1/3_Training Samples $\square$ 1/2_Training Samples $\Delta$ A All_Training Samples

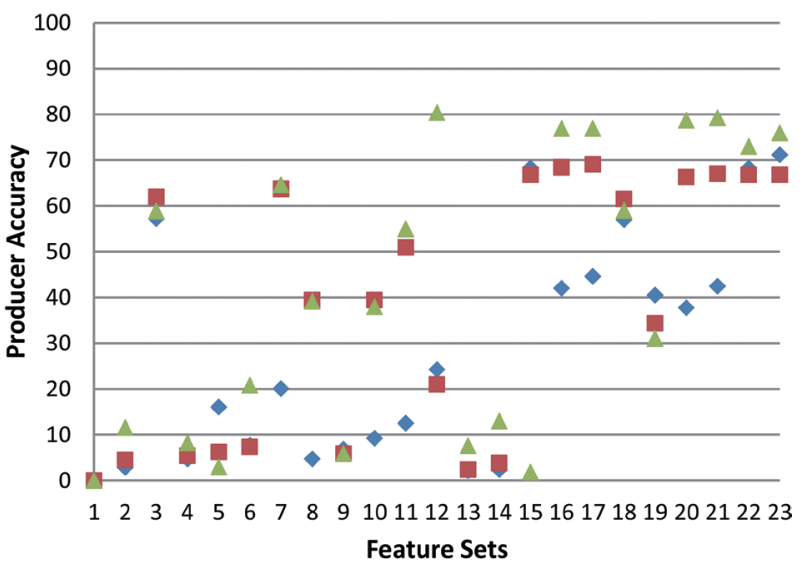

Fig. 8. The producer accuracy (PA) for each land-cover type. (a) PA for the broad-leaf forest; (b) PA for the grass; (c) PA for the bare land; (d) PA for the cement pavement; (e) PA for the built-up area. 
(a) $\$ 1 / 3 \_$Training Samples $\square$ 1/2_Training Samples $\Delta$ All_Training Samples

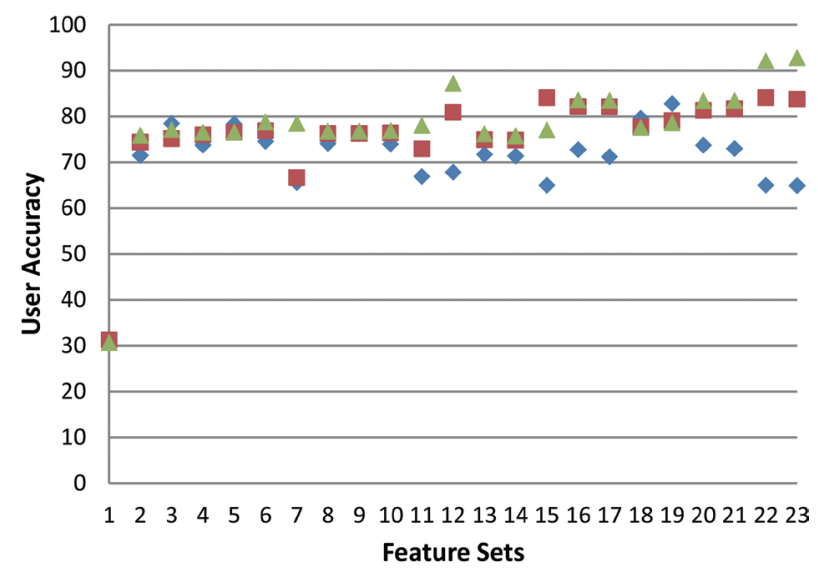

(c) $\$ 1 / 3 \_$Training Samples $\| 1 / 2$ _Training Samples $\Delta$ All_Training Samples

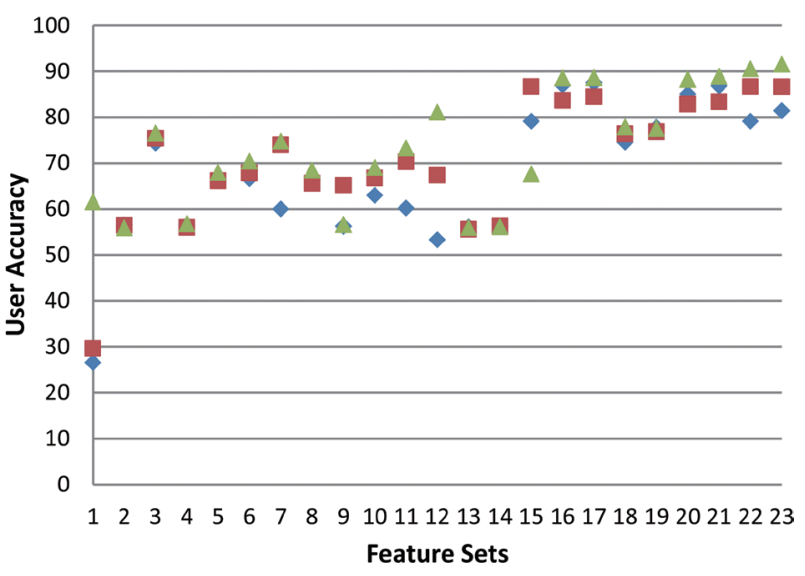

(e) $1 / 3_{-}$Training Samples $\square$ 1/2_Training Samples $\Delta$ All_Training Samples

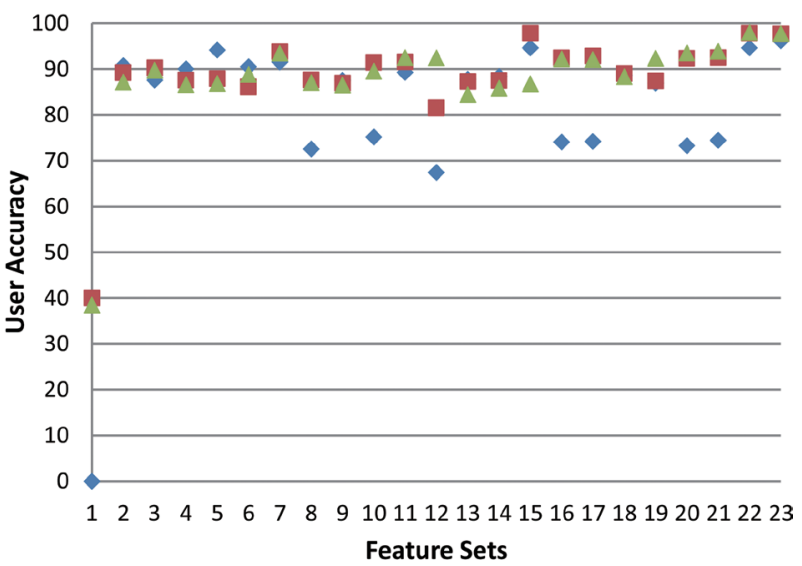

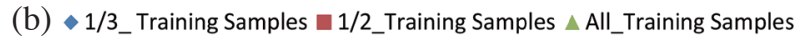

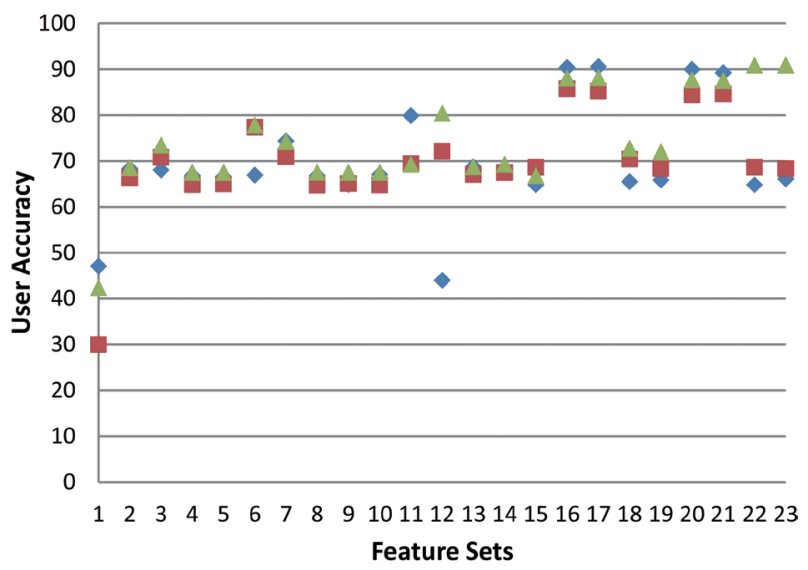

(d) 1/3_Training Samples $₫ 1 / 2 \_$Training Samples $\Delta$ All_Training Samples

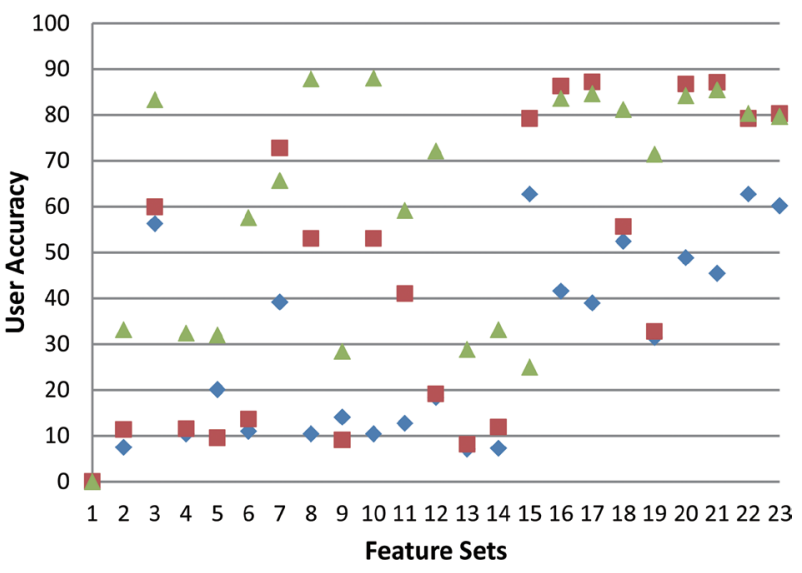

Fig. 9. The user accuracy (UA) for each land-cover type. (a) UA for the broad-leaf forest; (b) UA for the grass; (c) UA for the bare land; (d) UA for the cement pavement; (e) UA for the built-up area. 
broadleaf forest, both the PA and UA approached $90 \%$ when all features were applied. The results were acceptable only when the Gabor texture was used (PA and UA $>85 \%$ ). The accuracy for the grass areas was generally lower compared with that of the broadleaf forest areas in sets $16-17$ (PA $80 \%$, UA $87 \%$ ), and the accuracy was acceptable only when the Gabor texture used (PA and UA are higher than 80\%). Regarding the bare land areas, acceptable results were obtained (PA $>90 \%$ ) regardless of the combination of features or the size of the training samples. However, UA should be considered because sets 16 - 17 and 20 - 23 yielded superior UA results (close to 90\%). Regarding cement surfaces, the PA and UA were low because there were too few training samples in the uninhabited study area. Regardless of which combination of features was examined, acceptable PA and UA results (>90\%) were obtained for built-up areas, even when a low number of training samples was used. Both the PA and UA can be higher than $92 \%$ for the forest and builtup types when using amplitude and pulse width.

\section{CONCLUSION}

After integrating the spectral, geomorphometric, waveform and textural features obtained using high-resolution aerial orthophotos and FW LiDAR data, we assessed and summarized the accuracy of LCC to determine the waveform patterns for various land-cover types. The experimental results yielded the following:

(1) The highest overall accuracy ( $>90 \%)$ was obtained when all features were applied. Adding the geomorphometric features markedly improved the LCC accuracy. Moreover, the Gabor texture was a highly effective textural feature for improving the accuracy. Although the performance of the waveform characteristics failed to meet the anticipated outcomes, the research data provide valuable training samples for testing the effects of waveform features.

(2) Concerning each land-cover type, both the PA and UA results achieved adequate accuracy when all features were applied. Regarding the textural features, acceptable results were achieved when only the Gabor texture was used for the four defined land-cover types except cement surfaces. Both the PA and UA can be higher than $92 \%$ for the forest and built-up types by applying amplitude and pulse width. However, the poor classification results for the cement surfaces indicated that adequate training was difficult to obtain because the study area was an uninhabited volcanic island; thus, the island is categorized as a low-density development area.

(3) The waveform features were successfully used to conduct LCC. The statistical results indicate that multiple pulses were present in the waveforms for forested areas, whereas only one pulse appeared in the waveforms for other land-cover types. Moreover, the level of moisture content might have affected the waveform amplitude in the LiDAR data by reducing the reflection intensity. Further testing is necessary to evaluate how waveform and textural features affect LCC accuracy.

Acknowledgements This paper presents a part of research results supported by the National Science Council of Taiwan (Grant No.: NSC-102-2621-M-006-002-MY3).

\section{REFERENCES}

Alexander, C., K. Tansey, J. Kaduk, D. Holland, and N. J. Tate, 2010: Backscatter coefficient as an attribute for the classification of full-waveform airborne laser scanning data in urban areas. ISPRS J. Photogramm., 65, 423-432, doi: 10.1016/j.isprsjprs.2010.05.002. [Link]

Barlow, J., Y. Martin, and S. E. Franklin, 2003: Detecting translational Landslide scars using segmentation of Landsat ETM+ and DEM data in the northern Cascade Mountains, British Columbia. Can. J. Remote Sens., 29, 510-517, doi: 10.5589/m03-018. [Link]

Beraldin, J. A., F. Blais, and U. Lohr, 2010: Laser scanning technology. In: Vosselman, G. and H. G. Maas (Eds.), Airborne and Terrestrial Laser Scanning, Whittles Publishing, UK, 1-42.

Bischof, H., W. Schneider, and A. J. Pinz, 1992: Multispectral classification of Landsat-images using neural networks. IEEE Trans. Geosci. Remote Sensing, 30, 482490, doi: 10.1109/36.142926. [Link]

Bretar, F., A. Chauve, J. S. Bailly, C. Mallet, and A. Jacome, 2009: Terrain surfaces and 3-D landcover classification from small footprint full-waveform lidar data: Application to badlands. Hydrol. Earth Syst. Sci., 13, 1531-1544, doi: 10.5194/hess-13-1531-2009. [Link]

Camps-Valls, G. and L. Bruzzone, 2005: Kernel-based methods for hyperspectral image classification. IEEE Trans. Geosci. Remote Sensing, 43, 1351-1362, doi: 10.1109/TGRS.2005.846154. [Link]

Chang, K. T. and J. K. Liu, 2004: Landslide features interpreted by neural network method. The International Archives of the Photogrammetry, Remote Sensing and Spatial Information Sciences, Vol. XX, Part B7, International Society of Photogrammetry and Remote Sensing (ISPRS), Istanbul, Turkey, 574-579.

Chang, K. T., J. K. Liu, Y. M. Chang, and C. S. Kao, 2010: An accuracy comparison for the landslide inventory with the BPNN and SVM methods. Gi4DM 2010, Turino, Italy.

Chang, K. T., J. K. Liu, and C. I. Wang, 2012a: An objectoriented analysis for characterizing the rainfall-induced shallow landslide. J. Mar. Sci. Technol., 20, 647-656, doi: 10.6119/JMST-012-0430-2. [Link]

Chang, K. T., F. G. Yiu, J. T. Hwang, and Y. X. Lin, 2012b: Accuracy assessment of land use classification using hybrid methods. Proc. SPIE 8524, Land 
Surface Remote Sensing, 85241Q, Kyoto, Japan, doi: 10.1117/12.976844. [Link]

Chen, H. P., K. T. Chang, W. C. Hsu, J. K. Liu, and F. C. Yu, 2013: Characterizing land-cover type with LiDAR full waveform pattern. 2013 International Conference on Earth Observations and Societal Impacts (ICEO\&SI 2013), JUNE 23-25, Tainan, Taiwan.

Comber, A., P. Fisher, and R. Wadsworth, 2005: What is land cover? Environ. Plann. B, 32, 199-209, doi: 10.1068/b31135. [Link]

Doneus, M., C. Briese, M. Fera, and M. Janner, 2008: Archaeological prospection of forested areas using fullwaveform airborne laser scanning. J. Archaeol. Sci., 35, 882-893, doi: 10.1016/j.jas.2007.06.013. [Link]

Feddema, J. J., K. W. Oleson, G. B. Bonan, L. O. Mearns, L.E. Buja, G. A. Meehl, and W. M. Washington, 2005: The importance of land-cover change in simulating future climates. Science, 310, 1674-1678, doi: 10.1126/ science.1118160. [Link]

Foody, G. M. and A. Mathur, 2004: A relative evaluation of multiclass image classification by support vector machines. IEEE Trans. Geosci. Remote Sensing, 42, 1335-1343, doi: 10.1109/TGRS.2004.827257. [Link]

Galli, M., F. Ardizzone, M. Cardinali, F. Guzzetti, and P. Reichenbach, 2008: Comparing landslide inventory maps. Geomorphology, 94, 268-289, doi: 10.1016/j. geomorph.2006.09.023. [Link]

Huang, Y. T., K. H. Hsiao, J. C. Lin, and H. J. Su, 2007: A study on landuse classification using ortho-rectified aerial photographs and high resolution satellite images. Proceedings of Asia Conference on Remote Sensing (ACRS) 2007, Kuala Lumpur, Malaysia.

Hwang, J. T., K. T. Chang, and H. C. Chiang, 2011: Satellite image classification based on Gabor texture features and SVM. The 19th International Conference on GeoInformatics, ShangHai, China, 1-6, doi: 10.1109/ GeoInformatics.2011.5980774. [Link]

ITT, 2010: ENVI EX User's Guide, United State of America.

Jutzi, B. and U. Stilla, 2006: Range determination with waveform recording laser systems using a Wiener Filter. ISPRS J. Photogramm., 61, 95-107, doi: 10.1016/j. isprsjprs.2006.09.001. [Link]

Knabenschuh, M. and B. Petzold, 1999: Data post-processing of laser scan data for countrywide DTM production. In: Fritsch, D. and R. Spiller (Eds.), Photogrammetric Week '99, Wichmann Verlag, Heidelberg, 233-240.

Liao, S. R., 2013: DEM generation with full-waveform LiDAR data in vegetation area. Master Thesis, National Chengchi University, Taipei, Taiwan, 84 pp. (in Chinese)

Lillesand, T. M., R. W. Kiefer, and J. W. Chipman, 2004: Remote Sensing and Image Interpretation, $5^{\text {th }}$ edition,
John Wiley \& Sons, Inc., 763 pp.

Lin, Y. C. and J. P. Mills, 2010: Factors influencing pulse width of small footprint, full waveform airborne laser scanning data. Photogramm. Eng. Rem. S., 76, 49-59.

Liu, J. K., K. T. Chang, J. Y. Rau, W. C. Hsu, Z. Y. Liao, C. C. Lau, and T. Y. Shih, 2009: The geomorphometry of rainfall-induced landslides in Taiwan obtained by airborne LiDAR and digital photography. In: Ho, P. G. P. (Ed.), Geoscience and Remote Sensing, InTech, 115-132, doi: 10.5772/8305. [Link]

Mallet, C., F. Bretar, M. Roux, U. Soergel, and C. Heipke, 2011: Relevance assessment of full-waveform LiDAR data for urban area classification. ISPRS J. Photogramm., 66, S71-S84, doi: 10.1016/j. isprsjprs.2011.09.008. [Link]

Mücke, W., 2008: Analysis of full-waveform airborne laser scanning data for the improvement of DTM generation. Master Thesis, Vienna University of Technology, Vienna, Austria.

National Fire Agency, 2007: The official statistics report of national fire agency - Natural disaster statistics in Taiwan, Ministry of the Interior. Available at http://www. nfa.gov.tw/main/index.aspx. (in Chinese)

Neuenschwander, A. L., L. A. Magruder, and M. Tyler, 2009: Landcover classification of small-footprint, fullwaveform LiDAR data. J. Appl. Remote Sens., 3, 1-13, doi: 10.1117/1.3229944. [Link]

Pielke, R. A., 2005: Land use and climate change. Science, 310, 1625-1626, doi: 10.1126/science.1120529. [Link]

Thiel, K. H. and A. Wehr, 2001: Operational production of DTMs using ScaLARS, OEEPE, Stockholm.

Vapnik, V. N., 1998: Statistical Learning Theory, WileyInterscience, New York, 768 pp.

Vyas, V. S. and P. Rege, 2006: Automated texture analysis with Gabor filter. GVIP J., 6, 35-41.

Wagner, W., A. Ullrich, V. Ducic, T. Melzer, and N. Studnicka, 2006: Gaussian decomposition and calibration of a novel small-footprint full-waveform digitising airborne laser scanner. ISPRS J. Photogramm., 60, 100112, doi: 10.1016/j.isprsjprs.2005.12.001. [Link]

Wagner, W., M. Hollaus, C. Briese, and V. Ducic, 2008: 3D vegetation mapping using small-footprint full-waveform airborne laser scanners. Int. J. Remote Sens., 29, 1433-1452, doi: 10.1080/01431160701736398. [Link]

Wehr, A. and U. Lohr, 1999: Airborne laser scanning-an introduction and overview. ISPRS J. Photogramm., 54, 68-82, doi: 10.1016/S0924-2716(99)00011-8. [Link]

Zhu, G. and D. G. Blumberg, 2002: Classification using ASTER data and SVM algorithms; The case study of Beer Sheva, Israel. Remote Sens. Environ., 80, 233-240, doi: 10.1016/S0034-4257(01)00305-4. [Link]

Zurada, J. M., 1992: Introduction to Artificial Neural Systems, West Pub. Co., 163-248. 